\title{
On the effect of rheology on seasonal sea-ice simulations
}

\author{
Chi F. Ip, William D. Hibler, in and Gregory M. Flato \\ Thayer School of Engineering, Dartmouth College, Hanover, NH 03755, U.S.A.
}

\begin{abstract}
A generalized numerical model which allows for a variety of nonlinear rheologies is developed for the seasonal simulation of sea-ice circulation and thickness. The model is used to investigate the effects (such as the role of shear stress and the existence of a flow rule) of different rheologies on the ice-drift pattern and build-up in the Arctic Basin. Differences in local drift seem to be closely related to the amount of allowable shear stress. Similarities are found between the elliptical and square cases and between the Mohr-Coulomb and cavitating fluid cases. Comparisons between observed and simulated buoy drift are made for several buoy tracks in the Arctic Basin. Correlation coefficients to the observed buoy drift range from 0.83 for the cavitating fluid to 0.86 for the square rheology. The average ratio of buoy-drift distance to average model-drift distance for several buoys is 1.15 (square), 1.18 (elliptical), 1.30 (Mohr-Coulomb) and 1.40 (cavitating fluid).
\end{abstract}

\section{INTRODUCTION}

In a large-scale sea-ice model developed for numerical simulation of ice motion over a seasonal cycle, the rheology used can have a significant effect on the ice-thickness build-up and ice circulation. Plastic rheologies, relating ice deformation and thickness to the internal stress in the ice cover, with a normal flow rule, have been used to date in many seasonal dynamic-thermodynamic simulations (e.g. Pritchard and others, 1977; Hibler, 1979). These rheologies have proven useful in simulating discontinuous slip near the coast, at the same time providing reasonable velocity fields in the central Arctic Basin. However, as indicated by limited numerical sensitivity studies with different types of plastic yield curves, the amount of shear strength significantly affects the ice build-up and can possibly cause a stoppage of ice outflow through the Fram Strait. In addition, there is also the possibility that a rheology without a normal flow rule, such as the Mohr-Coulomb failure criterion used in soil mechanics, when used in a sea-ice model, may cause a different icevelocity pattern, especially in the Fram Strait region. To date no seasonal simulations with such non-normal flow rule rheologies have been performed.

The purpose of this study is to investigate the effect of different plastic rheologies on seasonal sea-ice circulation prediction. A generalized numerical scheme which allows simulation of the dynamic-thermodynamic behavior of sea ice with a variety of different non-linear rheologies is developed. Using such a numerical scheme, large-scale equilibrium simulations over a seasonal cycle are carried out with various plastic rheologies. This paper is divided into three sections. First, the basic features of the sea-ice model used are presented, focusing particularly on the four rheologies used: elliptical, square, cavitating fluid and Mohr-Coulomb. Then, the generalized numerical scheme is given with a discussion of the advantages and the disadvantages of the scheme. Next, some model simulation results are examined. Ice-velocity patterns and thickness build-up are compared along with a comparison between a few observed buoy tracks in the Arctic Basin and model-drift tracks. Finally, the results are analyzed to determine the effects of shear stress and flow rule on thickness build-up and ice circulation.

The overall goal of this study is to compare the effect of the different forms of the plastic rheology on sea-ice simulations. All the rheologies used are designed to have the same maximum ice strength, $P^{*}$, as a normalization parameter. This parameter is found to be crucial in ice rheology modeling (e.g. Hibler and Walsh, 1980). Other parameters used in the ice model, for example drag coefficients, are kept constant in order to single out the effect of the different shapes of yield curve. These other quantities may be used in future studies to fine tune the model for a better match of simulation results with observations.

\section{MODEL DESCRIPTION}

The numerical model developed in this investigation is a modification of the Hibler (1979) two level numerical model for the simulation of sea-ice circulation and thickness over a seasonal cycle. The main components of the model include: a momentum balance of various external, internal and inertial forces; a constitutive law which relates the ice stress to the strain rate and ice strength; a simple ice-thickness distribution which accounts for the change of ice thickness and concentration due to growth, advection and deformation; and an ice strength determined as a function of the ice thickness and compactness. 
A detailed description of the original model formulation and the physical aspects are given by Hibler $(1979,1980)$. Since it is the focus of the present study, a more detailed examination of the ice constitutive law and the plastic yield curves is given below.

\section{Constitutive law}

For modeling ice interaction, the ice is considered to be a non-linear viscous compressible fluid obeying the constitutive law

$$
\sigma_{i j}=2 \eta \dot{\epsilon}_{i j}+(\zeta-\eta) \dot{\epsilon}_{k k} \delta_{i j}-P \delta_{i j},
$$

where $\sigma_{i j}$ is the two-dimensional stress tensor, $\dot{\epsilon}_{i j}$ the strain-rate tensor, $P$ is a pressure term (taken as a function of ice-thickness characteristics), and $\zeta$ and $\eta$ are non-linear bulk and shear viscosities. The above equation, with an additional cross tensor term, is known as the Reiner-Rivelin model. The viscosities themselves are functions of the strain-rate invariants. Such a constitutive law enters the momentum balance of sea ice as an internal ice force. For calculations, the dependence of $\zeta$ and $\eta$ on $\dot{\epsilon}_{i j}$ and $P$ are given by a yield curve in principal stress space. The current study examines some of the effects yield curve shape has on ice flow. Figure 1 shows graphically the four plastic failure criteria used in the present study. Since the system is assumed to be isotropic, the principal stress and strain directions coincide. The normal flow rule states that, when plastic flow occurs (the stress state lies on the yield curve), the strain-rate vector is normal to the yield curve. Of the four different yield curves, three (elliptical, square and cavitating fluid) obey the normal flow rule, whereas the Mohr-Coulomb criterion does not.

\section{Elliptical yield curve}

The failure stress for this case lies on an ellipse passing through the origin with a no-stress condition applying for pure divergence. The equations for $\zeta$ and $\eta$ are

$$
\begin{aligned}
\zeta & =\frac{P^{*}}{2 \Delta}, \quad \eta=\zeta e^{-2} \quad \text { and } \quad(2 a, b) \\
\Delta^{2} & =\left(\dot{\epsilon}_{11}^{2}+\dot{\epsilon}_{22}^{2}\right)\left(1+e^{-2}\right)+4 \dot{\epsilon}_{12}^{2} e^{-2}+2 \dot{\epsilon}_{11} \dot{\epsilon}_{22}\left(1-e^{-2}\right),
\end{aligned}
$$

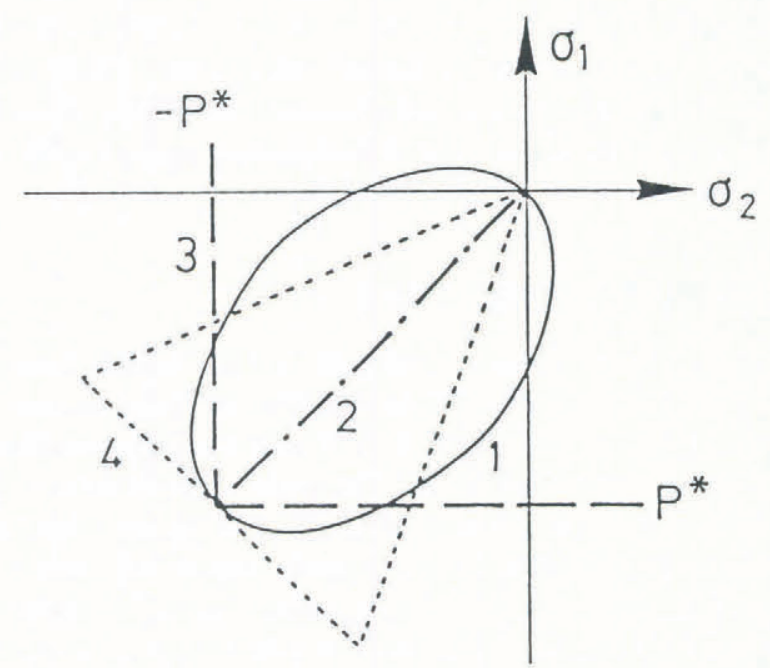

Fig. 1. Four plastic yield criteria in principal stress space: elliptical (1), cavitation fluid (2), square (3) and Mohr-Coulomb (4). with $e$ being the ratio of principal axes of the ellipse. Derivation of the above expression is given by Hibler (1977). Such a formulation assumes rigid plastic flow together with a normal flow rule. To prevent the values of the viscosities from becoming arbitrarily large (for small strain rates), $\zeta$ and $\eta$ are limited to ceiling values dependent on the ice strength $P^{*}$. When this occurs, the stress state lies on some smaller ellipse inside the yield surface. The ice cover under such small strain rates is assumed to move as a viscous fluid, or creep - an approximation to the desired rigid behavior. In the present model, the inside ellipse is positioned such that one of the vertices lies on the origin. This is in contrast to a concentric ellipse used in the Hibler (1979) model. The advantage of this formulation is that, in the absence of external forcing, there will be no motion. The differences in the formulation of the elliptical yield curve are not felt to be critical on the large scale and comparisons show that the two formulations of the elliptical yield curve give essentially the same result.

\section{The Mohr-Coulomb criterion}

The shear strength for this case is taken to be proportional to the compressive stress and the explicit $P$ dependence in the constitutive law is removed. Such a rheology does not have a normal flow rule but, rather, stress states on the two limbs of the Mohr-Coulomb yield curve correspond to pure shear deformation. A cap which limits the maximum allowable compressive stress is introduced to enable ice convergence. The cap strength is parameterized as a function of ice thickness and compactness. The included angle is chosen as $45^{\circ}$ for all long-term simulations, which corresponds to an angle of shear resistance, $\phi$ (in the usual soil mechanics notation), of about $25^{\circ}$. The bulk viscosity, $\zeta$, is zero for pure divergence, and

$$
\zeta=\min \left[\zeta_{\max }, \frac{P^{*}}{\left|\dot{\epsilon}_{1}+\dot{\epsilon}_{2}\right|}\right]
$$

otherwise. The shear viscosity, $\eta$, is given by

$$
\eta=\min \left[\zeta \tan \alpha\left|\frac{\dot{\epsilon}_{1}+\dot{\epsilon}_{2}}{\dot{\epsilon}_{2}-\dot{\epsilon}_{1}}\right|, \eta_{\max }\right] \text {, }
$$

where $\dot{\epsilon}_{\mathrm{j}}$ are the principal strain rates, $\alpha$ is the included angle, and $\zeta_{\max }$ and $\eta_{\max }$ are specified maximum viscosities. For the present study, the two maxima are taken to be the same as that for the elliptical yield curve. In addition, the cap for the Mohr-Coulomb cap case is located at the maximum extent of the elliptical yield curve. Such a failure criterion with no normal flow rule has been used in soil mechanics, but it has not been used in seasonal ice simulations to date.

\section{Cavitating fluid}

The cavitating fluid yield curve in principal stress space is that of the Mohr-Coulomb failure criterion with a zero included angle, so no shear stress exists. In this case the normal flow rule is also satisfied. The dependence of $\zeta$ is the same as in the Mohr-Coulomb case with $\alpha$ taken to be zero and the shear viscosity, $\eta$, also taken to be zero. The cavitating fluid rheology is of interest mainly for its lack of shear stress. 


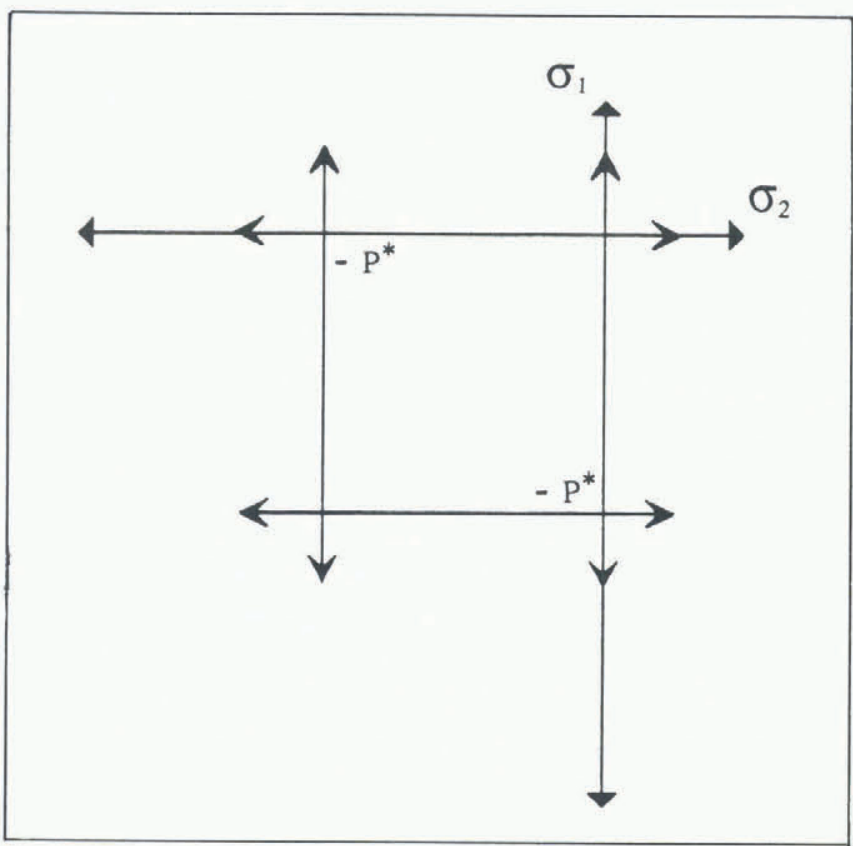

Fig. 2. Strain-rate vectors and plastic stress state correspondences for square rheology.

\section{Square rheology}

The plastic stress state for this case is confined to four corners of a square. By limiting plastic flow to the vertices, such a yield curve does obey an associative normal flow rule. Figure 2 shows the correspondence between the strain-rate vector and the four failure points. Note that stress-free states correspond to pure divergence. This rheology has the useful physical interpretation that there is a fixed compressive failure stress along any convergent principal axis. For the square yield curve, the value of $\zeta$ is not determined explicitly. Instead a bulk viscosity enters Equation (1) as a pressure term $P$ (the first stress invariant) such that

$$
P=-\frac{\sigma_{1}+\sigma_{2}}{2}=-\zeta\left(\dot{\epsilon}_{1}+\dot{\epsilon}_{2}\right),
$$

where $P$ is

$$
\begin{aligned}
P=\min \left[\frac{P^{*}}{2}\right. & \left., \zeta_{\max }\left|\min \left(\dot{\epsilon}_{1}, 0\right)\right|\right] \\
& +\min \left[\frac{P^{*}}{2}, \zeta_{\max }\left|\min \left(\dot{\epsilon}_{2}, 0\right)\right|\right] .
\end{aligned}
$$

The dependence of $\eta$ is given as

$$
\eta=\min \left(\eta_{\max }, \eta_{\mathrm{c}}\right),
$$

where $\eta_{\mathrm{c}}$ is

$$
\begin{aligned}
& \eta_{\mathrm{c}}=\frac{P^{*}}{\left|\dot{\epsilon}_{1}-\dot{\epsilon}_{2}\right|}, \quad \text { if } \quad P \leq \frac{P^{*}}{2}, \\
& \eta_{\mathrm{c}}=\frac{P^{*}-P}{\left|\dot{\epsilon}_{1}-\dot{\epsilon}_{2}\right|}, \quad \text { if } \quad P>\frac{P^{*}}{2}
\end{aligned}
$$

$\zeta_{\max }$ and $\eta_{\max }$ are specified values of viscosity. When

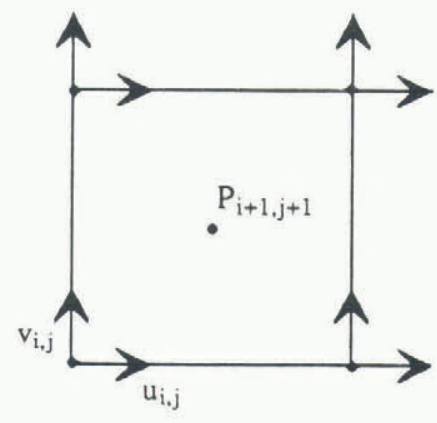

Arakawa B-Grid

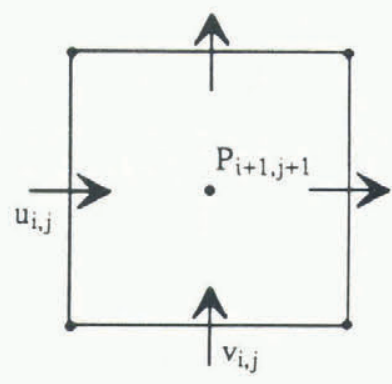

Arakawa C-Grid
Fig. 3. Comparison between computation BGrid and C-Grid.

either of the maximum values are used, the ice is considered to be in a creep state. When this occurs, the stress state lies either on the side of the square or inside. For comparison purposes, the state of pure convergence is located at the maximum extent of the elliptical yield case.

\section{NUMERICAL SCHEME}

The numerical scheme used in the present investigation differs from the Hibler (1979) ice model in two aspects: (1) location of ice velocity points in each grid, (2) ice dynamic calculation procedure.

\section{Computational grid}

The present numerical scheme uses the Arakawa C-Grid geometry instead of the B-Grid of the Hibler (1979) model. The difference between these grids is illustrated in Figure 3. Here, scalar quantities for both cases lie at the center of the cell. For the B-Grid, the ice velocities are placed at the corners, but for the C-Grid they are located on the sides. The reason for using the C-Grid configuration is two-fold. Firstly, the C-Grid allows for a simpler finite differencing formulation for most of the terms in the internal force calculation. Secondly, by using the C-Grid, an alternating grid point instability in the pressure field is avoided and the total kinetic energy fluctuation with time is also eliminated.

\section{Ice dynamics calculation}

The time-stepping numerical scheme replaces the relaxation routine for the momentum time step in the Hibler (1979) model with an explicit forward Euler timestepping scheme. A similar technique, using a rheology exhibiting bulk viscosity only, was used by Semtner (1987). Such a technique allows direct calculation of the stress states and the ice forces once a specific yield curve is given. It is the use of such a method which allows the treatment of more generalized yield curves with ease. However, while more general, the procedure is less computationally efficient than any semi-implicit procedure. For the present scheme to be numerically stable, the maximum time step is of the order of seconds as opposed to the one-day time step for the Hibler (1979) model. Figure 4 contrasts the total kinetic energy evolution for both a stable and an unstable case, demonstrating the importance of time-step selection. 


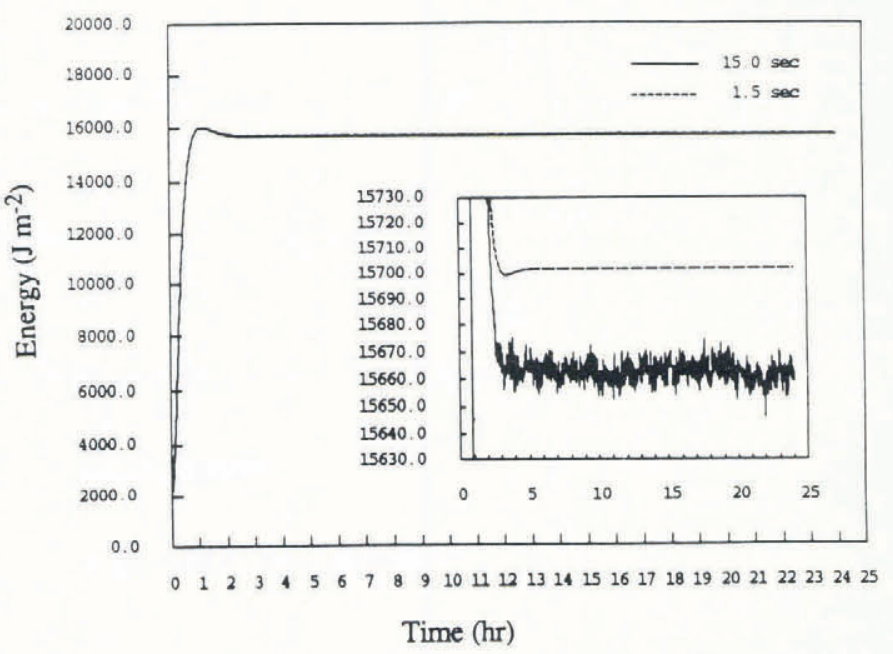

Fig. 4. Time-series of total kinetic energy for stable and unstable time step. The two curves are results for $1.5 \mathrm{~s}$ and $15.0 \mathrm{~s}$ time steps using the Mohr-Coulomb rheology. The inset shows the same time-series with an expanded scale so that the oscillatory nature of the unstable solution can be seen.

Table 1. Computation requirement and stability time-step limit for different rheologies

\begin{tabular}{lcc}
\hline Rheology & $\begin{array}{c}\text { CPU d }^{-1} \\
(\mathrm{~min})\end{array}$ & $\begin{array}{c}\text { Time-step limit } \\
(\mathrm{s})\end{array}$ \\
\hline Square & 22.70 & 4.50 \\
Cavitating fluid & 46.81 & 2.75 \\
Elliptical & 103.97 & 1.25 \\
Mohr-Coulomb* & 105.28 & 1.25 \\
\hline
\end{tabular}

* The time-step limit here is shown for an included angle, $\alpha$, of $45^{\circ}$.

From the time-stepping point of view, Table 1 provides a listing of the time-step stability limit for the four rheologies using the present numerical scheme. Included in the table is the CPU time required for a one-day simulation on an Alliant FX-40 mini-supercomputer. For the present computer code, the computational time required on the Alliant supercomputer is comparable to the time required using a Cyber 205. Note that, whereas the square rheology is least constrained in terms of the time step, it is still computationally inefficient compared to the relaxation method of Hibler (1979). In the MohrCoulomb yield curve case, the matter is further complicated by the time-step limit dependence on the included angle, $\alpha$. The amount of shear stress allowable in the Mohr-Coulomb criterion is parameterized by the included angle: when $\alpha$ equals zero, the caviating fluid is obtained; when $\alpha$ equals $90^{\circ}$, a portion of the square yield curve is obtained. Figure 5 shows graphically the dependence of time-step limit on the value of $\alpha$. From the figure, it appears that the time-step limit is reduced dramatically with an increase in the value of the included angle (or amount of shear stress allowed at failure).

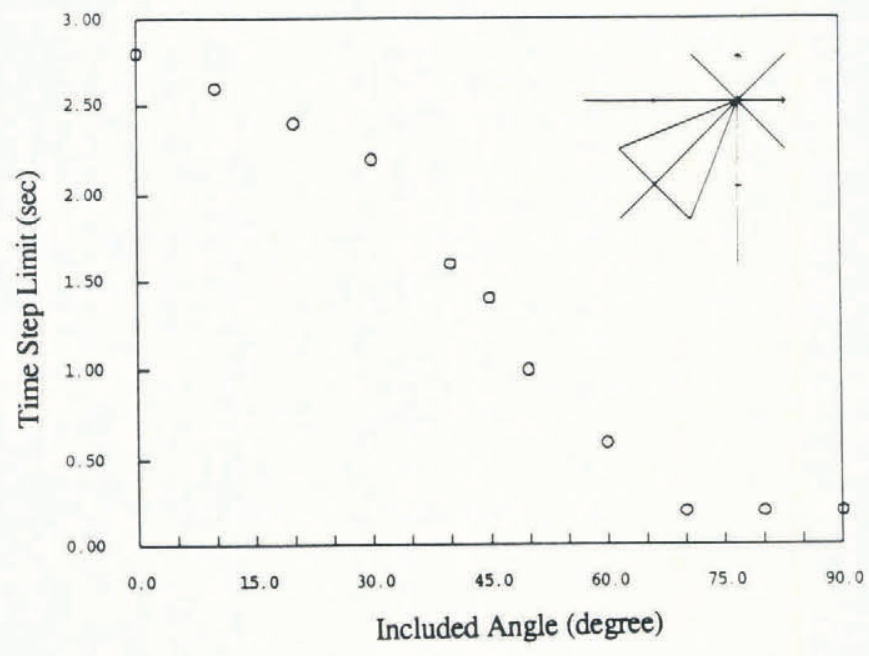

Fig. 5. Stability requirement (included angle dependence) for Mohr-Coulomb yield curve.

As indicated, the explicit numerical scheme, although useful, is extremely computationally intensive due to small time-step requirement. The actual CPU time required for a one-year integration varies from $28 \mathrm{~h}$ for the square rheology to about $56 \mathrm{~h}$ for the Mohr-Coulomb case. For a study of the rheological effects on sea-ice simulations, it is desirable to use other less computationally intensive methods if available. Other more efficient relaxation methods do exist for the elliptical, cavitating fluid and Mohr-Coulomb rheologies. The time required for a one-year integration for these models ranges from 1 to $3 \mathrm{~h}$. The three faster models are: Hibler (1979) model for elliptical case; Flato and Hibler (1990) cavitating fluid model for cavitating fluid case; and Flato and Hibler (1990) model for Mohr-Coulomb case (Flato and Hibler, 1991). The last method is an extension of the cavitating fluid model with added shear stress in which the finite differencing scheme for handling the shear components is identical to that of the explicit time-stepping method described here. Note that the square rheology cannot be readily implemented with a relaxation scheme. The comparison of direct time-stepping implementation of the various rheologies to the more efficient relaxation schemes is briefly discussed below.

\section{Comparison of relaxation method with explicit time-stepping scheme}

Before proceeding to the long-term simulations, some short-term simulations will be discussed to compare the two numerical schemes. One-day simulations with a constant initial thickness field were carried out for the elliptical, cavitating fluid and Mohr-Coulomb rheologies for both the explicit time-stepping procedure and the relaxation methods. The resulting ice-velocity pattern and pressure field were compared and found to be very similar. Furthermore, particle trajectories were also calculated and compared. Here, several initial starting points on the grid were chosen and the particle paths were traced for $300 \mathrm{~d}$ using the one-day velocity field result. In general, the magnitude of the vector difference between the daily drift paths calculated using the two different numerical schemes did not exceed $1 \%$. Thus, the use of the more efficient relaxation method instead of current 
explicit time-stepping procedure for long-term simulation of elliptical, Mohr-Coulomb and cavitating fluid for the present study is justified.

In addition, for the explicit time-stepping scheme, the one-day simulation results were also used to determine the locations of the stress states in principal space. It was found that about $70-80 \%$ of the states for the entire grid lie on the corresponding yield curves. Thus, the explicit time-stepping procedure does indeed seem to simulate plastic flow.

\section{SIMULATION RESULTS}

All the simulations discussed below were performed on a grid representing the Arctic Ocean with $160 \mathrm{~km}^{2}$ grid cells as shown in Figure 6. Here the cross hatches indicate outflow boundary points.

\section{Boundary and initial conditions}

For the long-term simulations discussed below, the models were forced with daily wind, surface air temperature and relative humidity provided by Walsh (personal communication) on a $5^{\circ} \times 5^{\circ}$ grid for $1981-82$, and mean annual ocean currents and heat flux from the diagnostic ice-ocean calculation of Hibler and Bryan (1987). For the square rheology, the time step used in the simulation is $4.5 \mathrm{~s}$ with a single day distorted so that $5 \mathrm{~h}$ is taken to be one day. Numerical experiments have been carried out to show that after $5 \mathrm{~h}$ the ice velocity has nearly reached the end of day value with the total kinetic energy reaching $99.5 \%$ of the end of day value. The time distortion reduced computer run time for one-year integration to about $30 \mathrm{~h}$. The maximum bulk viscosity is reduced by a factor of five from the Hibler (1979) model value to improve the stability of the explicit time-stepping method. One-day time steps are used for all the other models. It is also important to note that all models used in the study have the same thermodynamic treatment as that of Hibler and Walsh (1980), which is essentially that of

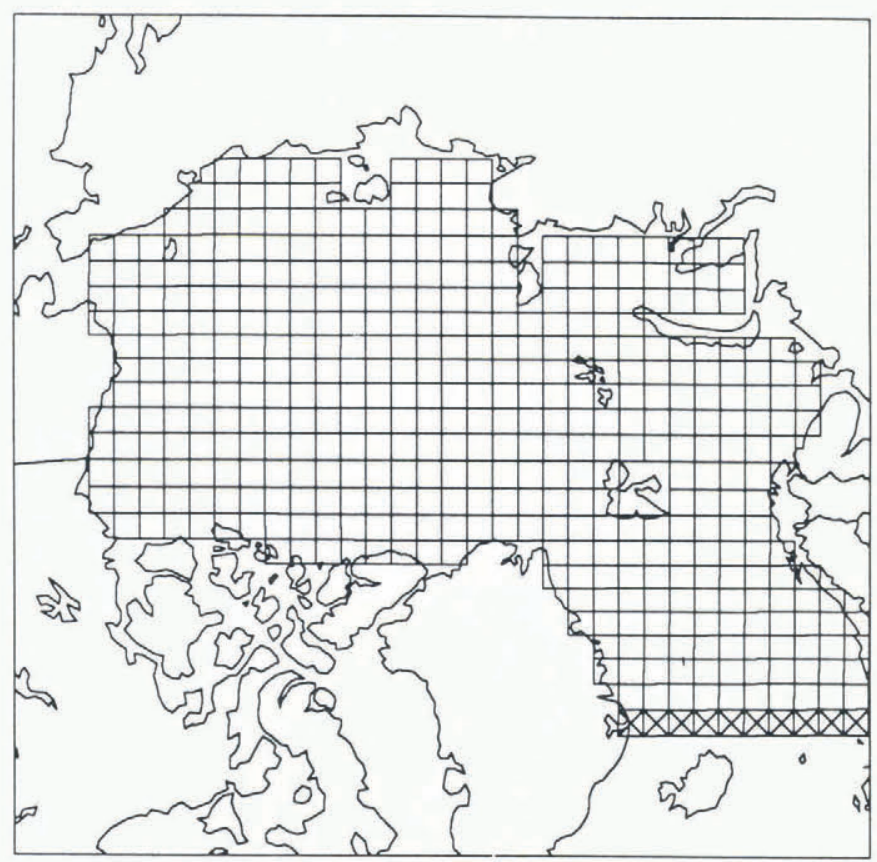

Fig. 6. Computation mesh used in simulations.
Parkinson and Washington (1979) with minor modifications.

\section{Velocity and ice-thickness build-up comparison}

In order to investigate the effect of different rheologies, the models were applied to the Arctic Basin and integrated for two years. Four separate simulations were carried out, each with a different ice rheology: (a) square, (b) elliptical, (c) Mohr-Coulomb with a cap and (d) cavitating fluid.

Monthly velocity fields were compared for all four cases and found to follow a similar pattern. Figure 7 shows the average monthly velocity pattern for March 1982 for all the different rheologies. It is noted that, for the time period studied, the square and elliptical rheologies produce a velocity field similar to one another as do the Mohr-Coulomb and cavitating fluid rheologies. However, there are differences when the results are compared between the two sets (elliptical and square to Mohr-Coulomb and cavitating fluid). Examining closely Figure $7 \mathrm{a}-\mathrm{d}$, it is noted that the velocity patterns for the Mohr-Coulomb and cavitating fluid cases reveal a small gyre near the North Pole which is absent for both the square rheology and the elliptical cases. In addition, the magnitudes of ice velocities for both the elliptical and square cases are found to be lower in the coastal regions especially near the Canadian archipelago. To show the importance of ice interaction, Figures 7e and $f$ show the case of an incompressible Mohr-Coulomb (Mohr-Coulomb failure with no cap) and for free drift (no ice interaction). Notice that the incompressible MohrCoulomb rheology produces an entirely different velocity field. On the other hand, free drift produces a velocity pattern similar to the other four rheologies studied, but it allows considerable convergence near the coast in contrast to actual observation. Note also that the icevelocity magnitude is greater for the free drift case, as expected.

The velocity averaged over each month and over the entire Arctic Basin, and the monthly average outflow through the Fram Strait, are compared next for the four rheologies. The basin velocity and the outflow through the Fram Strait averaged over a two-year period (198182) for the different rheologies is listed in Table 2. Again similarities exist for the elliptical and square cases and for the cavitating fluid and Mohr-Coulomb model. It is found that both the cavitating fluid and Mohr-Coulomb cases result in higher velocities than the other two cases. The same holds true for the outflow through the Fram Strait. The smaller velocity and outflow for the elliptical and the square yield curves are believed to be a result of the greater shear stress that these two cases allow.

Spatial patterns of ice-thickness build-up for each month of the two-year integration were also examined for the four cases and were found to be very similar. Consequently no significant effect of rheology could be ascertained. However, total Arctic Basin ice mass (volume) did differ. Figure 8 gives the time series of total ice volume retained in the Arctic Basin for 1982. Here, it is seen that a greater thickness build-up occurs for the square and elliptical rheologies. This result appears to be due to the greater ice outflow through Fram Strait for the Mohr-Coulomb and cavitating fluid rheologies (Table 2). 

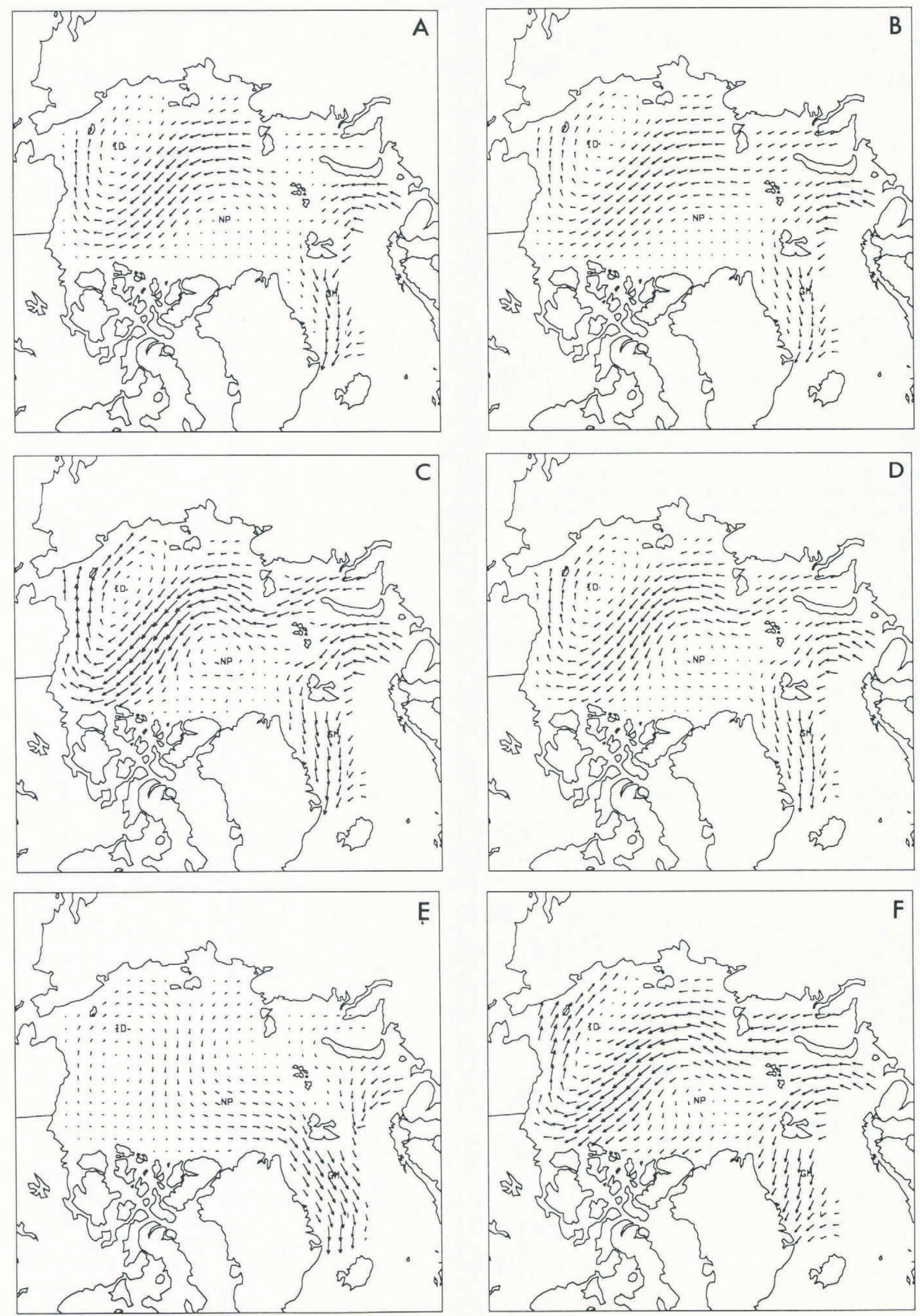

Fig. 7. Average monthly velocity fields for March 1982: (a) elliptical; (b) square; (c) MohrCoulomb; (d) cavitating fluid; (e) incompressible Mohr-Coulomb; (f) free drift. A velocity vector one grid cell long is approximately $0.12 \mathrm{~m} \mathrm{~s}^{-1}$. 
Table 2. Arctic Basin ice velocity and outflow through the Fram Strait averaged over the period 1981-82 for different rheologies

\begin{tabular}{lcc}
\hline Rheology & $\begin{array}{c}\text { Average basin velocity } \\
\left(\mathrm{m} \mathrm{s}^{-1}\right)\end{array}$ & $\begin{array}{c}\text { Average outflow } \\
(\mathrm{Sv})\end{array}$ \\
\hline Square & 0.0435 & 0.0767 \\
Elliptical & 0.0426 & 0.0558 \\
Mohr-Coulomb & 0.0534 & 0.0891 \\
Cavitating fluid & 0.0602 & 0.0932 \\
$\begin{array}{l}\text { Incompressible } \\
\quad \text { Mohr-Coulomb }\end{array}$ & 0.0425 & 0.1007 \\
\hline
\end{tabular}

Table 3. Correlation coefficients between monthly observed and monthly averaged ice drift for different rheologies. The statistics are based on the six buoy tracks shown in Figure 9

\begin{tabular}{lc}
\hline \multicolumn{1}{c}{ Rheology } & Correlation coefficient \\
\hline Square & 0.863 \\
Mohr-Coulomb & 0.852 \\
Elliptical & 0.839 \\
Cavitating fluid & 0.829 \\
Incompressible Mohr-Coulomb & 0.827 \\
Free drift & 0.825 \\
\hline
\end{tabular}

* All results are calculated based on monthly values.

Table 4. Comparison of modeled and observed buoy-drift statistics for different rheologies. The average radius is the average magnitude of the vector difference between the observed and simulated monthly drift. The model drift-buoy drift is the ratio of model predicted drift to average monthly buoy-drift distance

\begin{tabular}{lcc}
\hline Rheology & $\begin{array}{c}\text { Average error radius } \\
(\mathrm{km})\end{array}$ & $\begin{array}{c}\text { Model drift/ } \\
\text { buoy drift }\end{array}$ \\
\hline Square & 69.58 & 1.15 \\
Incompressible & & 1.17 \\
$\quad$ Mohr-Coulomb & 74.95 & 1.18 \\
Elliptical & 75.87 & 1.30 \\
Mohr-Coulomb & 76.07 & 1.33 \\
Free drift & 81.71 & 1.39 \\
Cavitating fluid & 88.07 & \\
\hline
\end{tabular}

* All results are calculated based on monthly values.

\section{Buoy-drift comparison}

Comparison of the overall drift patterns was treated in a previous section. This section will deal with some comparison of observed and simulated buoy drift. Several observed buoy-drift tracks in the Arctic Basin are shown in Figure 9. These buoy-drift data were obtained from World Data Center A for Glaciology and are based on buoy data collected by the Arctic Buoy Program (Colony, personal communication). The lines here connect the monthly observed locations of six individual buoys beginning at June 1981. Figure 10 shows a close-up of two

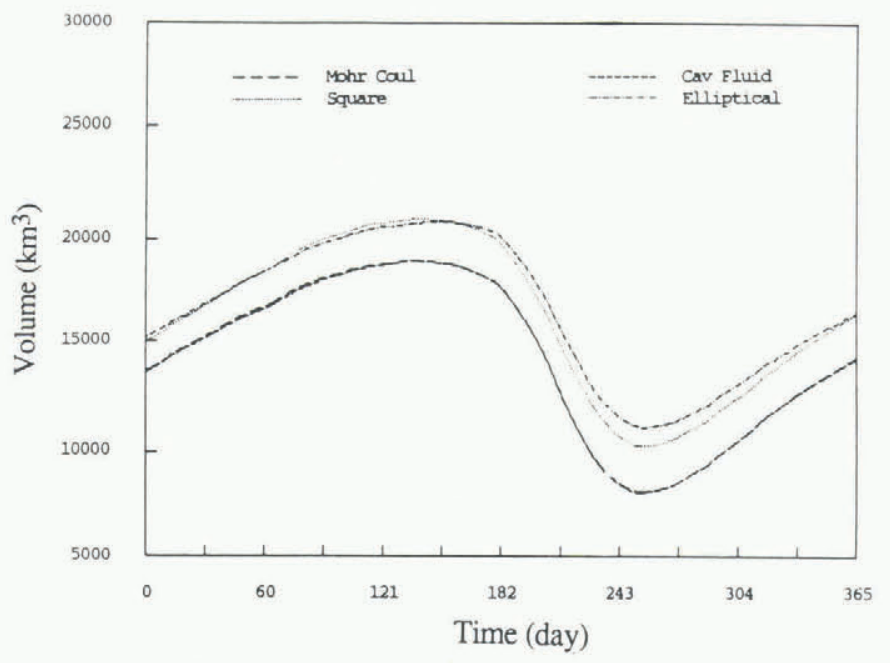

Fig. 8. Total ice volume retained in the Arctic Basin for 1982.

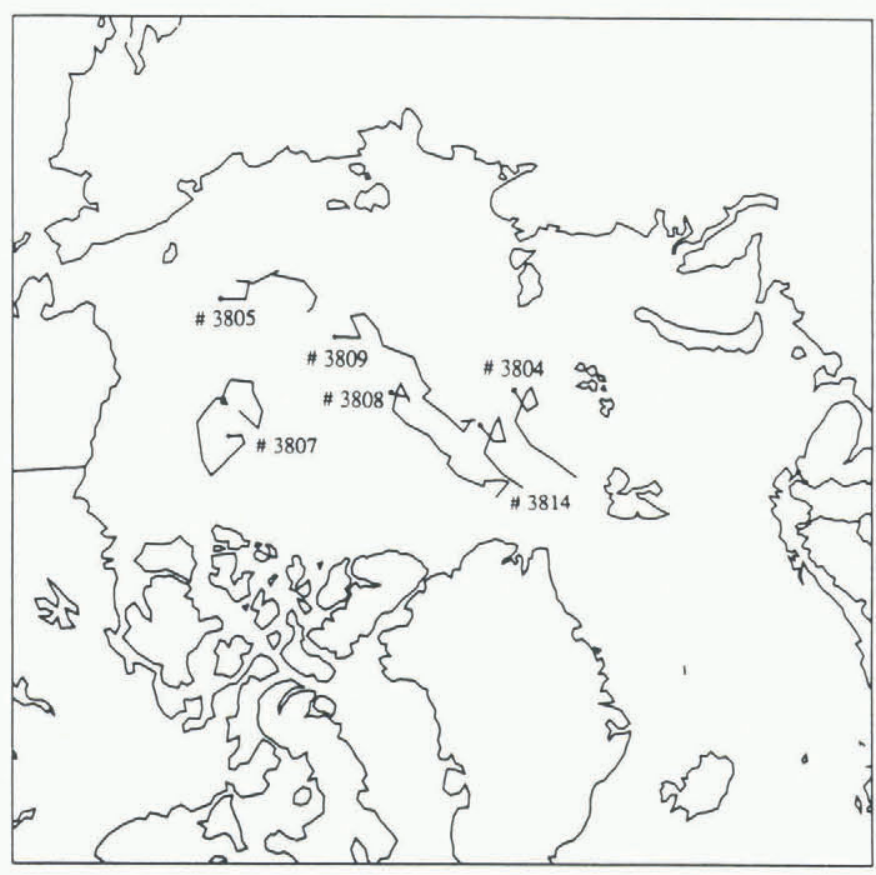

Fig. 9. Observed buoy-drift tracks in the Arctic Basin. The buoys all begin to drift at June 1981.

particular buoy tracks along with predicted tracks for the different rheologies involved. Here, the location of the observed buoy was first identified and the corresponding velocity predicted from the model was calculated for a month. The resulting monthly drift vectors were then added vectorally. The model drift shown is thus a cumulative drift track. There are two points to notice in Figure 10. First, the buoy tracks calculated for the elliptical and square rheologies tend to cluster together as do the Mohr-Coulomb and cavitating fluid. Second, the square and elliptical cases exhibit slower drift compared to the other two cases. Such a slow-down may be caused by the larger allowable shear stress present in the former rheologies. On the same figure, predicted buoy drifts for the incompressible Mohr-Coulomb and free drift are shown on the right. Here, the free drift case exhibits the largest drift distance whereas drift in the incompressible case is considerably reduced. The clustering of the el- 

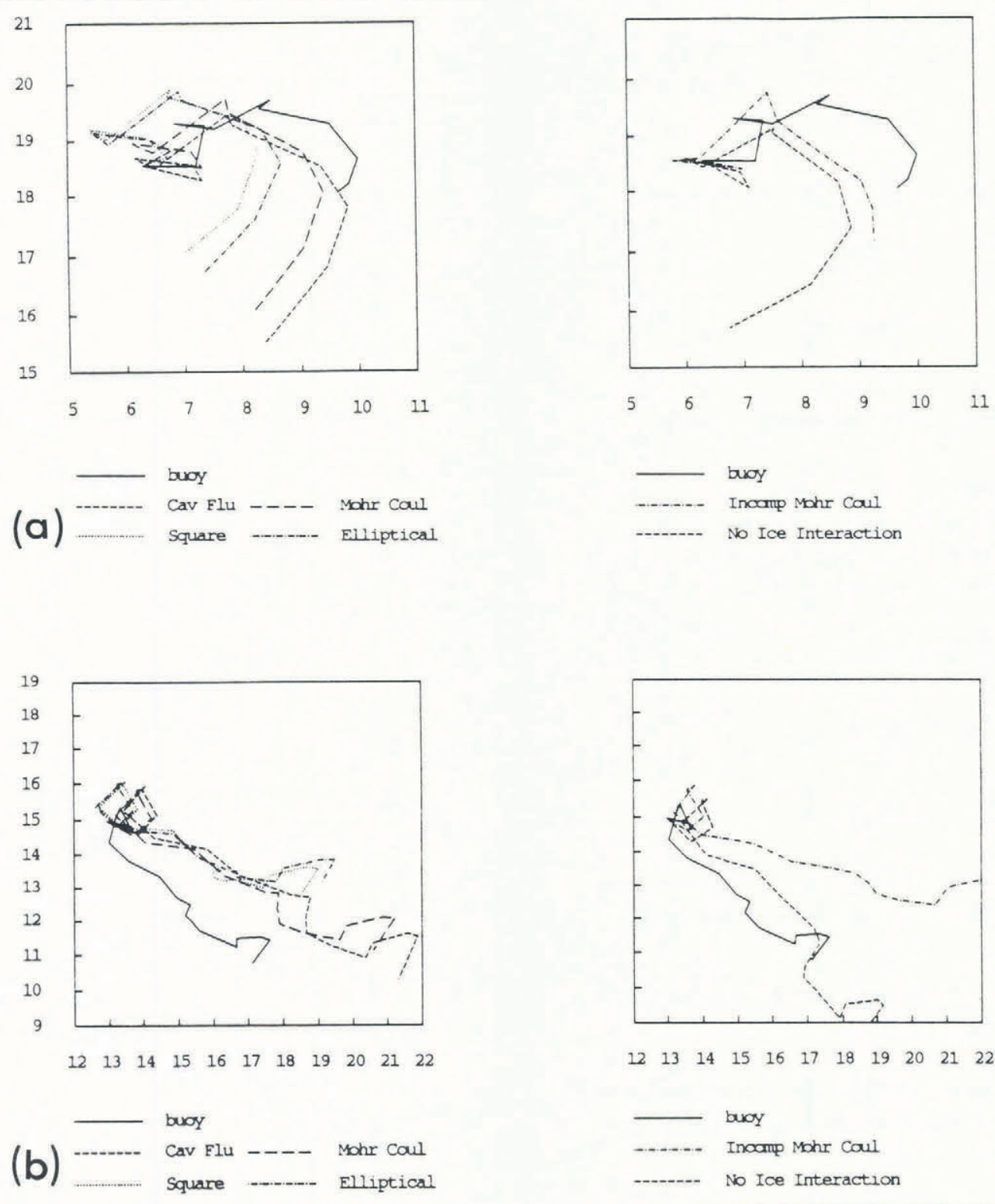

$\begin{array}{lllllllllll}12 & 13 & 14 & 15 & 16 & 17 & 18 & 19 & 20 & 21 & 22\end{array}$

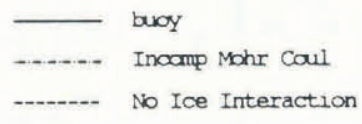

Fig. 10. Comparison of observed and simulated buoy drift. The axes are in terms of grid cell coordinates. (a) Buoy no. 3805. (b) Buoy no. 3808.

liptical and square cases and of the cavitating fluid and Mohr-Coulomb cases supports the earlier comparison of velocity pattern and ice-thickness build-up.

In terms of statistical analysis, correlation coefficients between monthly observed and computed drift were calculated based on the six buoy tracks studied. These correlation coefficients are shown in Table 3 . We see here that the square rheology is the best correlated with the observed buoy drift whereas the free drift case is the worst. However, considering the limited data, the coefficients are too close for all the rheologies to distinguish between them. Table 4 presents some further buoy-drift statistics. In this case the average error radius is the average magnitude of the vector difference between the observed and computed monthly drift. The model-drift ratio to buoy-drift ratio is the ratio of model predicted drift to average monthly buoy-drift distance. Again, the values reported are not significantly different among the rheologies although the square rheology is the best case.
It is believed that a better comparison of rheologies lies in examination of velocity pattern, thickness build-up, and individual model predicted drift tracks. In addition, statistical results and drift comparison based on daily values may be more useful than the monthly average values used in this study.

\section{CONCLUSIONS}

A general time-stepping numerical scheme was developed which allows a variety of non-linear sea-ice rheologies to be simulated in the context of a seasonal sea circulation model. While useful for short-term comparisons, the computational effort required to obtain a truly stable solution limits this model's utility for long-term simulations. More efficient implicit numerical schemes were compared to the general method for several idealized rheologies and were found to be satisfactory for the studies conducted here. From the present investigation, it is 
seen that large-scale sea-ice plastic rheology has a significant effect on long-term simulations of ice drift; however, the effect on thickness build-up patterns is surprisingly small. In particular, the Mohr-Coulomb and cavitating fluid rheologies produce a much greater ice drift (compared to both the other rheologies and observed data) because of the lower shear strength. It appears that the Mohr-Coulomb and cavitating fluid rheologies used here may be less realistic than the elliptical and square cases due to the low shear strength. The velocity pattern of the incompressible Mohr-Coulomb case demonstrates that the type of plastic failure criterion used can be crucial in sea-ice simulations.

For the four plastic rheologies examined in this study, similarities in both velocity pattern and ice-thickness build-up exist between the elliptical and the square case and between the Mohr-Coulomb and cavitating fluid. Some of the similarities between the Mohr-Coulomb and cavitating fluid model may be explained by the fact that pure shear deformation is required for all stress states except zero and the compressive strength limit. The situation is different in the square and elliptical cases, wherein shearing deformation occurs at intermediate stress states. The similarity between the elliptical and square rheologies may also be explained in part by the similarity of the shear stress to compressive stress ratio. It was also shown that the amount of allowable shear stress does play a role in sea-ice simulations. This is evident in the lower average basin velocity and outflow through the Fram Strait observed for both the elliptical and square yield surfaces where a large shear is possible for small compressive stress.

The cumulative buoy-drift comparison supported the findings regarding the resemblance between the elliptical and square and between Mohr-Coulomb and cavitating fluid. However, monthly buoy-drift statistics were found to be inadequate in distinguishing between the rheologies. Daily buoy-drift data may be more helpful in this case and are currently being investigated.

\section{ACKNOWLEDGEMENT}

This work was supported by the U.S. Office of Naval Research under contract N00014-86-K-069.

\section{REFERENCES}

Flato, G.M. and W.D. Hibler, III. 1990. On a simple sea-ice dynamics model for climate studies. Ann. Glaciol., 14, 72-77.

Flato, G.M. and W. D. Hibler, III. In press. On modeling pack ice as a cavitating fluid. J. Phys. Oceanogr.

Hibler, W.D., III. 1977. A viscous sea ice law as a stochastic average of plasticity. J. Geophys. Res., 82(27), 3932-3938.

Hibler, W.D., III. 1979. A dynamic thermodynamic sea ice model. J. Phys. Oceanogr., 9(4), 815-846.

Hibler, W.D., III. 1980. Documentation for a twolevel dynamic thermodynamic sea ice model. CRREL Spec. Rep. 80-8.

Hibler, W.D., III, and K. Bryan. 1987. A diagnostic iceocean model. J. Phys. Oceanogr., 17(7), 987-1015.

Hibler, W.D., III, and J.E. Walsh. 1982. On modeling seasonal and interannual fluctuations of Arctic sea ice. J. Phys. Oceanogr., 12(12), 1514-1523.

Parkinson, C.L. and W.M. Washington. 1979. A largescale numerical model of sea ice. J. Geophys. Res., 84(C1), 311-337.

Pritchard, R.S., M.D. Coon and M.G. McPhee. 1977. Simulation of sea ice dynamics during AIDJEX. J. Press. Vessel Technol., 99, 491-497.

Semtner, A.J., Jr. 1987. A numerical study of sea ice and ocean circulation in the Arctic. J. Phys. Oceanogr., 17(8), 1077-1099.

The accuracy of references in the text and in this list is the responsibility of the authors, to whom queries should be made. 